\title{
Unravelling complex molecular mechanisms in pine with genome-wide association analysis and gene co-expression networks
}

\author{
Stephanie Karenina Bajay, Alexandre Hild Aono, Matias Kirst, and Anete Pereira de \\ Souza
}

The authors have withdrawn this manuscript because they decided to change the phenotypic characteristics to be evaluated, considering the more significant contribution to the field. Therefore, the authors do not want this work to be cited as a reference for the project. If you have any questions, please contact the corresponding author. 